\title{
Chemical Separation on Silver Nanorods Surface Monitored by TOF-SIMS
}

\author{
Ondrej Petruš, ${ }^{1}$ Andrej Oriňak, ${ }^{1}$ Renáta Oriňaková, ${ }^{1}$ Christian Muhmann, ${ }^{2}$ Ján Macko, ${ }^{1,3}$ \\ Radim Hrdý, ${ }^{4}$ Jaromír Hubálek, ${ }^{4}$ Branislav Erdelyi, ${ }^{1,5}$ and Heinrich F. Arlinghaus ${ }^{2}$ \\ ${ }^{1}$ Faculty of Sciences, Department of Physical Chemistry, University of P. J. Šafárik in Košice, Moyzesova 11, 04154 Košice, Slovakia \\ ${ }^{2}$ Westfälische Wilhelms-Universität Münster, Physikalisches Institut, Münster, Germany \\ ${ }^{3}$ Faculty of Science, Department of Physical Chemistry, Comenius University, Mlynská Dolina II, Bratislava, Slovakia \\ ${ }^{4}$ SIX Center, Faculty of Electrical Engineering and Communication, Brno University of Technology, Technická 3058/10, \\ 31600 Brno, Czech Republic \\ ${ }^{5}$ Institute of Physics, Faculty of Sciences, University of P. J. Šafárik in Košice, Park Angelinum, 04154 Košice, Slovakia
}

Correspondence should be addressed to Andrej Oriňak; andrej.orinak@upjs.sk

Received 28 December 2016; Accepted 10 April 2017; Published 1 June 2017

Academic Editor: Victor David

Copyright (C) 2017 Ondrej Petruš et al. This is an open access article distributed under the Creative Commons Attribution License, which permits unrestricted use, distribution, and reproduction in any medium, provided the original work is properly cited.

The article introduces a possible chemical separation of a mixture of two compounds on the metal nanorods surface. A silver nanorods surface has been prepared by controlled electrochemical deposition in anodic alumina oxide (AAO) template. Rhodamine 6G and 4-aminothiophenol have been directly applied to the sampling point on a silver nanorods surface in an aliquot mixture. The position of the resolved compounds was analysed by time-of-flight secondary ion mass spectrometry (TOF-SIMS) which measured the fragments and the molecular ions of the two compounds separated on the silver nanorods surface. Rhodamine $6 \mathrm{G}$ has been preconcentrated as $1.5 \mathrm{~mm}$ radial from the sampling point while 4 -aminothiophenol formed a continuous self-assembled monolayer on the silver nanorods surface with a maximum molecular ion intensity at a distance of $0.5 \mathrm{~mm}$ from the sampling point. The separation of the single chemical components from the two-component mixture over the examined silver nanostructured films could clearly be shown. A fast separation on the mentioned nanotextured films was observed (within $50 \mathrm{~s}$ ). This procedure can be easily integrated into the micro/nanofluidic systems or chips and different detection systems can be applied.

\section{Introduction}

Recent advances in material research proves novel strategies finally resulting in sophisticated surface morphology for chemical separation based on molecular patterning or self-assembly. Bases of these processes result from spontaneous molecular interactions to control the particle size, shape, or other surface characteristics of the assembly resulting in a highly ordered and oriented unique structure. Very important are these molecular assemblies that may possess nanoscale features as well as novel properties that macroscale materials often lack. These structures are highly ordered and oriented nanotubes, nanocavities, nanowires, nanoposts, nanocones, nanospheres, molecular imprints, nanoparachutes (conical monodendrons), and general nanoparticle surfaces with random structures, often [1].

Nanopatterned structures offer a high surface to volume ratio and specific interaction on molecular level as well as possibility of applying direct micro/nanodetection of the separated analytes. Pioneer research in this area was documented in previous articles where $10 \mu \mathrm{m}$ thick monolithic silica sorbent layer gives faster separation with lower limits of detection and reduced analyte and solvent volumes. Produced were ultrathin layers on chromatography plates with a controllable nanostructure and thickness. Experiments showed that the layer separation characteristics depended on the film nanostructure [2]. In another article [3] the $\mathrm{SiO}_{2}$ nanopillars were used as scaffolds for functionalization with 
octadecyltrichlorosilane to generate a superhydrophobic stationary phase in liquid chromatography. A further progress in nanostructure based separation media or layer development has been reported in [4]. Nanopatterned surfaces (nanopillars) and nanoparticles have been already applied in electrophoretic and electrochromatographic separation [5]. A nanopatterned film has been prepared by molecular imprinting and used in electrophoretic separation. The separation of a liquid to multiple layers on a nanopillars surface represents a phenomenon with future application in chip technology [6].

An excellent example of separation ability of the nanotextured films mentioned a novel strategy to reversibly tune the pore size of nanotube membranes as they are mechanically compressed and released [7]. The membrane was molecule size-selective ( 5 to $20 \mathrm{~nm}$; applied in proteins separation) based on the compression level and exhibited excellent backwardness (elastically cycled hundreds of times without any significant reduction in the membrane thickness) and was easily cleaned. Mentioned application is belonging to miniaturized systems and chips. The integration of nanostructured films into microchannels is known to enable unique and useful separation in both electrophoresis and chromatography. Etched pillars and other nanostructures have received considerable interest in recent years as a platform for creating microchannels with pores tailored to specific applications. Glancing angle deposition was used to fabricate nanostructures that were subsequently embedded in PDMS microchannels using a sacrificial resist process. Demonstrated was application of chiral and chevron nanostructures. This versatility in structural design could facilitate new developments in on-chip separation [8].

A coupling of the nanopatterned layers technology and chip level systems for pressure driven liquid chromatography has been already documented [9]. New media for nanoseparation present multiwalled carbon nanotubes (MWCNTs). They can be easily synthesized directly in microfluidic channel and integrated in separation chips [10]. However, recent progress was achieved by integrating functional nanostructured films to nanofluidics. They require strong driving forces for liquid transport and depend on bulky accessory equipment like pumps and various control devices as well as others [11]. One example of how to overcome these problems would be to use directly an adenosine- $5^{\prime}$-triphosphate- (ATP) driven biological molecular motor for the nanoseparation and concentration of analyte molecules directly onto the detector positions [12]. The nanopatterned film can pose in many functions; documented was example of silver surface with two functions: chemical separation and detection. A combination of active surface enhanced Raman spectroscopy (SERS) nanostructured surfaces within micro/nanofluidics could create a perfect sensing platform for trace chemicals detection with several advantages including fingerprinting ultrasensitivity, portability, minimum use of samples/reagents, and generation waste, as well as faster reaction times [13]. Several applications of the molecular ratchet in the separation science have already been mentioned [14]. In general, there are two types of molecular ratchet: one type is much like a rectifying nanostructure where movement is easier in one direction of highly ordered and oriented nanoparticles (nanopillars) than the other because a larger force is necessary for one direction compared to the other. This type of molecular ratchet uses an oscillating timeaveraged zero force as its input (e.g., an E-field or a pressure gradient), which rectifies molecules of mixture [14]. The other type is more subtle and applies a periodic change of the height of the molecular ratchet nanopillar or nanorod (or other shape of nanoparticle) [15]. This type of molecular ratchet uses Brownian motion as its input and switches from the state where the energy barriers (ratchet nanoparticle) are lower than the thermal energy $\mathrm{kT}$ to a state where they are higher. Unfortunately, all analytical Brownian molecular ratchets produced up until now had micrometre-highly ordered structures and the separation performance was not yet comparable with classical methods $[16,17]$.

By the physical separation on nanotextured films the living cells can be entrapped. Different adhesion of them on a nanostructure allows trapping cancer cells from blood $[18,19]$.

In this article a new multifunctional silver nanorods film with proposed chemical separation ability is introduced. The separation function of silver nanorods surface has been confirmed by TOF-SIMS that monitored position of the solutes on silver nanorods surface.

\section{Experimental}

2.1. Chemicals. All chemicals were of analytical grade and all solutions were prepared freshly. The chemicals were used without further purification. $(\mathrm{COOH})_{2}, \mathrm{CrO}_{3}, \mathrm{H}_{3} \mathrm{PO}_{4}$, and $\mathrm{AgNO}_{3}$ were purchased from Sigma-Aldrich (Germany) and 4-aminothiophenol (4-AMTP) and rhodamine 6G (R6G) from Alfa Aesar (Germany).

2.2. TOF-SIMS Analysis. TOF-SIMS measurements were performed using an ION-TOF IV compatible instrument equipped with a $25 \mathrm{kV} \mathrm{Bi}_{3}{ }^{+}$liquid metal ion gun (IONTOF GmbH, Germany). For the experiments the following parameters were used: $200 \mu$ s cycle time, $0.05 \mathrm{pA}$ primary ion current, and no electron flooding. High mass resolution spectra were acquired from different areas of $150 \mu \mathrm{m} \times$ $150 \mu \mathrm{m}$. The mass/charge ratios of these secondary ions were measured with a time-of-flight mass spectrometer to determine the elemental, isotopic, or molecular composition of the silver nanorods surface.

2.3. Preparation of Alumina Oxide Membrane Templates. The silicon wafer was coated with a tungsten layer with a thickness of $200 \mathrm{~nm}$ and subsequently with a high-purity thin aluminum layer $(99.99+\%)$ with a thickness of $150 \mathrm{~nm}$ by thermal evaporation (PVD). The thin porous anodic alumina oxide (AAO) templates were prepared by using equipment consisting of a flow system controlled by a membrane pump, a power source, a thermostat, and a personal computer which provided the automatic driving of all processes. The AAO templates were prepared by a one-step anodic oxidation of aluminium thin film under constant voltage of $40 \mathrm{~V}$ 


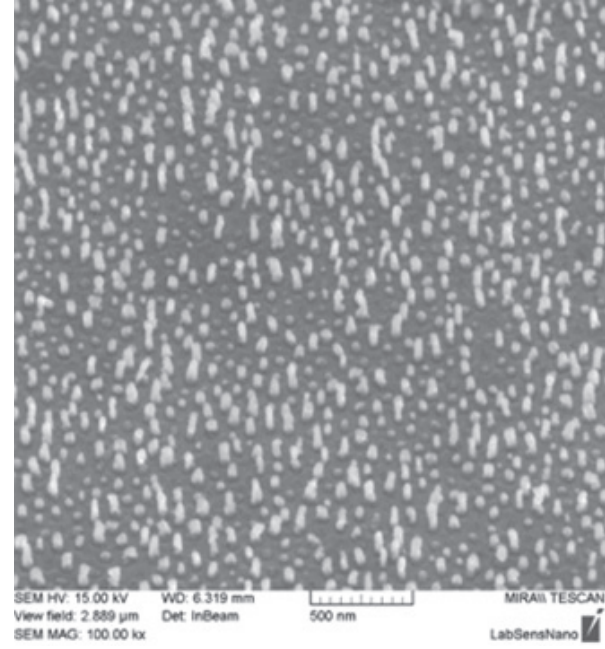

FIGURE 1: SEM micrograph of the silver nanorods film prepared by electrochemical deposition.

in $0.3 \mathrm{~mol} / \mathrm{L}$ oxalic acid solution in a conventional twoelectrode system at $16^{\circ} \mathrm{C}$ for $180 \mathrm{~s}$. A gold wire was used as counter electrode. Vertically aligned pores were formed by the honeycomb structure in the alumina layer. Afterwards, a high current droop was detected indicating that the Al layer was completely transformed to $\mathrm{AAO}$ and $\mathrm{WO}_{3}$ nanodots, which were obtained on the $\mathrm{W}$ and AAO interface below each pore. Thereafter, the sample was washed by distilled water and dried. This technique of AAO fabrication has been described in more detail in recent paper [20].

\subsection{Preparation of the Silver Nanostructures. Electrochemical} pulse deposition was used to fabricate the silver nanorods into the nanoporous AAO template. Deposition was performed from $0.2 \mathrm{~mol} / \mathrm{dm}^{3} \mathrm{AgNO}_{3}\left(\mathrm{pH} 2.5\right.$ ) at $60^{\circ} \mathrm{C}$ in two subsequent steps consisting of 20 deposition pulses with a current intensity of $1 \mathrm{~mA}$ and a duration of $2 \mathrm{~s}$. The electrolyte was allowed to circulate on the AAO template surface for 10 minutes before the pores of the membrane were filled. The current pulses were applied using an Autolab PGSTAT $302 \mathrm{~N}$ potentiostat with a standard two-electrode setup. A thin gold layer prepared by evaporation on AAO membrane was used as a cathode and the platinum electrode as an anode. A conducted tungsten underlayer was used as a cathode. Finally, the surface was etched in aqueous solution containing $5 \mathrm{~mL}$ of $85 \% \mathrm{H}_{3} \mathrm{PO}_{4}$ and $3 \mathrm{~g} \mathrm{CrO}_{3}$ for $6 \mathrm{~s}$ at a temperature of $45^{\circ} \mathrm{C}$ to remove the AAO. A SEM micrograph of the resulting nanostructured silver layer is shown in Figure 1. The depicted nanosized structure consisted of the nanorods with a diameter of $50 \mathrm{~nm}$ and a height of $150 \mathrm{~nm}$.

\section{Results and Discussion}

$1 \mu \mathrm{L}$ of the mixture $(1: 1)$ containing $10^{-4}$ molar water solution of R6G and $10^{-4}$ molar water solution of 4-AMTP was deposited on the surface of the selected silver nanorods substrate. After the evaporation of the sample liquid (solvent),

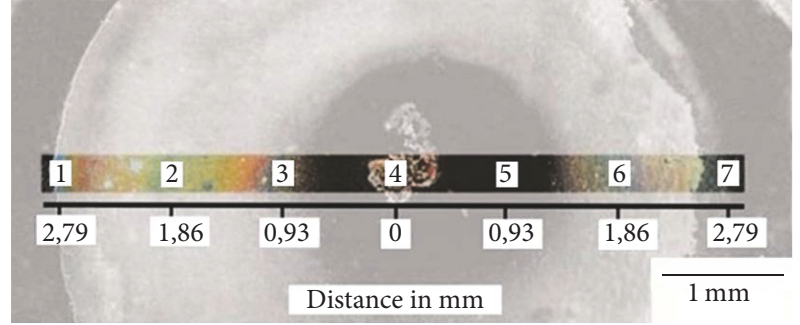

FIGURE 2: Schematic overview of the seven positions which were imaged with TOF-SIMS. The sampling point was in position 4 . The picture clearly shows the radial sample separation. The dimensions were measured with a goniometer.

the sample was analysed with TOF-SIMS. TOF-SIMS images were obtained in the direction from the center to layer border and from different positions from the left and right site of the spot using step sizes of approximately $1 \mathrm{~mm}$ (Figure 2). Molecular ion masses for both R6G and 4-AMTP $(\mathrm{m} / z=$ $401, m / z=125)$ analytes were taken for reconstructing high-resolution TOF-SIMS images. The chemical separation on the silver nanorods film was determined with TOF-SIMS by using the above-mentioned molecular ions as well as the masses $m / z$ 80, 93, and 97 for additional confirmation. No mass interferences were observed for the analytes fragment masses. The TOF-SIMS image depicted in Figure 3 shows different distributions for the two molecular analyte ions on the silver nanopatterned film. The TOF-SIMS molecular ions abundances clearly show that 4-AMTP stays more retained at the surface than R6G, which is eluted as the first analyte followed by 4-AMTP. Moreover, 4-AMTP forms on the silver nanotextured film a self-assembled monolayer (SAM) as it has already been documented in the earlier published article [21]. Then, R6G moves over 4-AMTPSAM; thus, the new nanoscale separation phase can affect R6G resulting in a retention on the silver nanorods. TOFSIMS images confirmed the R6G preconcentration in $1.5 \mathrm{~mm}$ distance from the sampling point (Figure 3, position 2). The identification of the position of 4-AMTP is more complicated due to SAM formation. A detailed TOF-SIMS analysis of the 4-AMTP fragment ions $m / z$ 80, 93, and 97 (Figure 4) showed a maximum abundance for $m / z 93$ (phenyl ion) in $0.5 \mathrm{~mm}$ distance from the sampling point. Both compounds in the mixture that were applied in the sampling point were separated on the silver nanorods (Figure 5). The separation mechanism is unknown. By using formal equations from chromatography, the retention factor $h R_{F, i}$ of each analyte separated has been calculated based on [22]

$$
h R_{F, i}=100 \cdot \frac{z_{i}}{z_{f}},
$$

where $z_{i}$ is the distance from sampling point to maximum abundance of ion $\mathrm{m} / z 401$ or $\mathrm{m} / z 125 ; z_{f}$ is the distance from sampling point to the solvent front elution distance. For R6G it was 0.54 and for 4-AMTP 0.18. The sample separation effectivity is affected by the type of nanostructure, sample viscosity, solvent evaporation rate, and surface energy. An 
Field of view $99.6 \times 99.6 \mu \mathrm{m}^{2}$
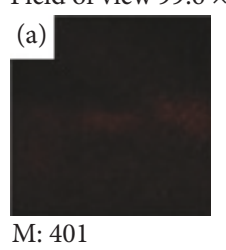

Tc: 10099
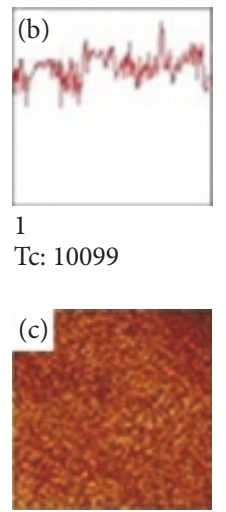

M: 125

Tc: 99956

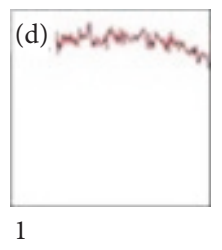

Tc: 99956

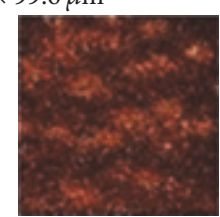

M: 401

Tc: 30515

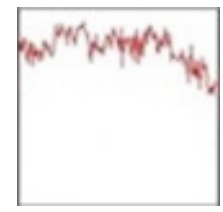

2
Tc: 30515

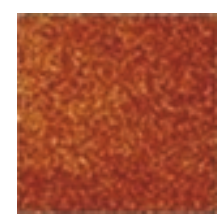

M: 125

Tc: 114813

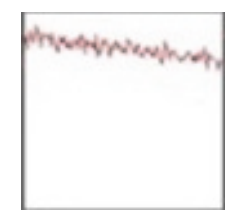

2

Tc: 114813

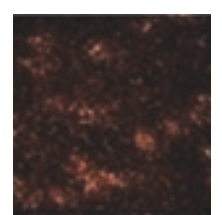

M: 401

Tc: 10231

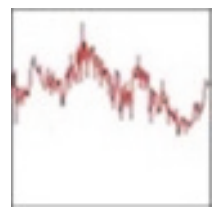

3

Tc: 10231

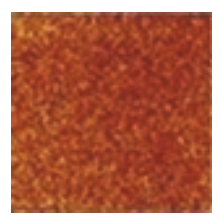

M: 125

Tc: 115177

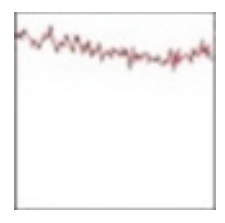

34

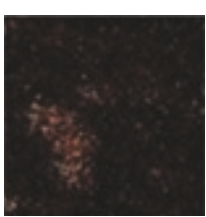

M: 401

Tc: 3198

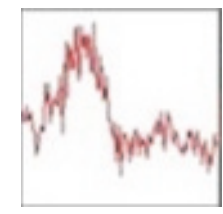

4

Tc: 3198

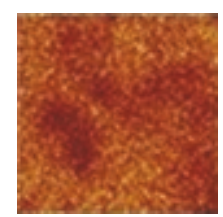

M: 125

Tc: 140415

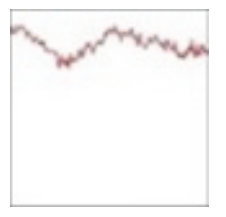

4
Tc: 140415

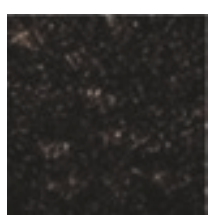

M: 401

Tc: 1954

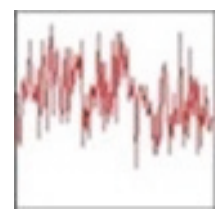

5

Tc: 1954

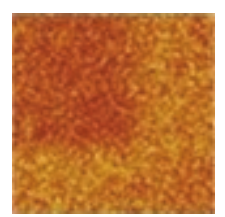

M: 125

Tc: 237592

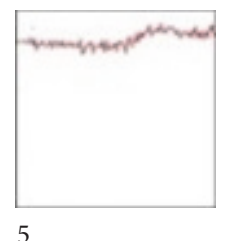

5

Tc: 237592

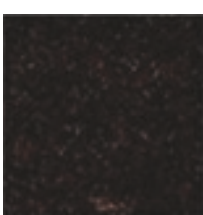

M: 401

Tc: 3052

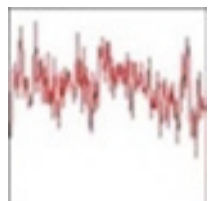

6

Tc: 3052

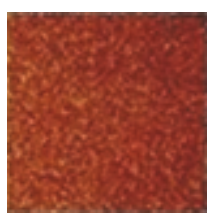

M: 125

Tc: 91104

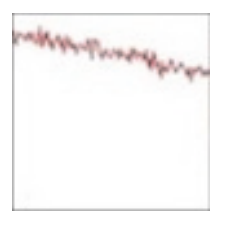

6

Tc: 91104

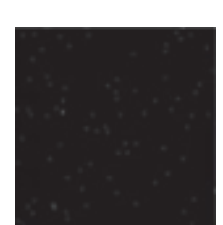

M: 401

Tc: 85

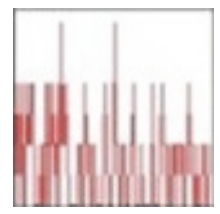

7

Tc: 85

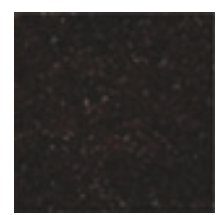

M: 125

Tc: 2681

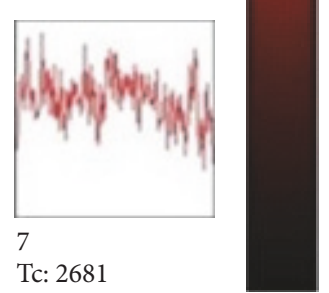

Figure 3: TOF-SIMS images and the line scans obtained at positions 1-7 shown in Figure 2. The TOF-SIMS signals from the line scans were obtained by summing up the measured counts for each image. Tc: total counts. (a) positive TOF-SIMS ion $m / z 401$ images distribution in substrate diameter intersection; (b) TOF-SIMS signal intensities distribution via the surface of the same sample intersection; (c) positive TOFSIMS ion $\mathrm{m} / \mathrm{z} 125$ images distribution in substrate diameter intersection; (d) TOF-SIM signal intensities distribution via the same sample intersection.

average liquid velocity on the surface can be expressed by modified Young equation (2) [23]:

$$
v_{C}=\frac{r_{c} \gamma \cos \theta}{4 z_{f} \eta},
$$

where $r_{c}$ is capillary diameter (calculated from nanostructure morphology), $\gamma$ is surface tension (at nanostructure), $\theta$ is contact angle, $\eta$ is viscosity, and $z_{f}$ is front distance of sample liquid.

The measured values of nanostructure surface contact angle were in the interval from $70^{\circ}$ to $110^{\circ}$. Separation can run by different separation modes at the same nanostructured surface. It represents the next feature in application of the nanostructured surfaces. Moreover, repeatedly dropping liquid to the sample point (by dosing device) as it is known from chromatography can improve separation and preconcentration of the analytes. The separation on silver nanorods is fast and efficient (within $50 \mathrm{~s}$ ). It behaves similar to thin-layer chromatography; however, the solute migration is directed by surface nanotexture and solvent evaporation rate. This causes some inhomogeneity in separation zone leading to zone spreading. Some separation specific, nanotextured films, preparation methods have already been documented in the articles published by Linford research group [24-27]. In the next step various PDMS microfluidic channel designs based on hydrogel procedure preparation will be examined [28] followed by an integration of nanostructured functional films into miniaturized systems. Functional nanostructured films can be incorporated into a channel by different ways: PDMS internal channel walls modification [29], electrochemical deposition, enzymatic reaction, embedding, self-assembled monolayers, and so forth. The final product can pose as a nanodevice with incorporated nanodetector, nanoseparation unit, filtration element, and liquid phase transport in one direction.

\section{Conclusions}

Surface functionalization and control over nanostructured interfaces represent a key aspect in nanoscale functionality. The separation ability of a new silver nanorods film was 


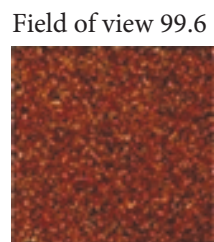

M: 80

1
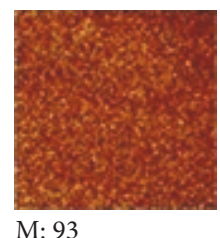

1

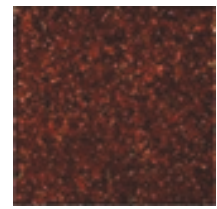

M: 97

1

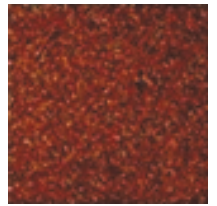

M: 80

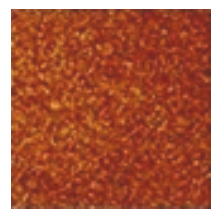

M: 93

2

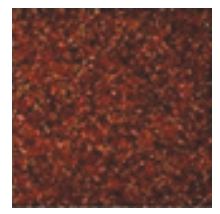

M: 97

2

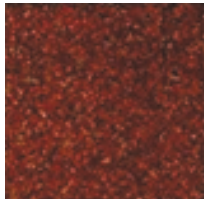

M: 80

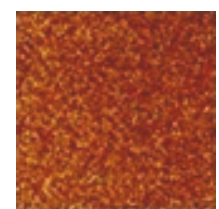

M: 93

3

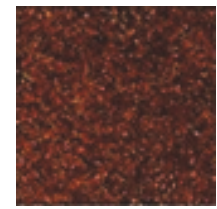

M: 97

3

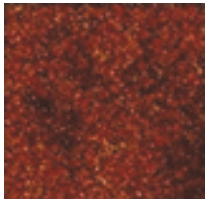

M: 80

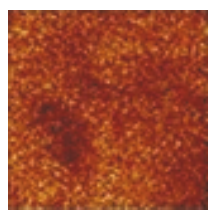

M: 93

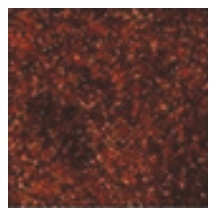

M: 97

4

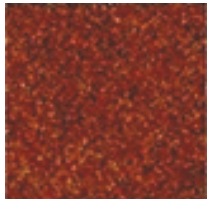

M: 80

5

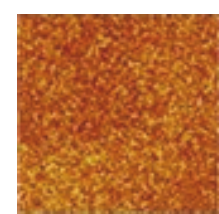

M: 93

5

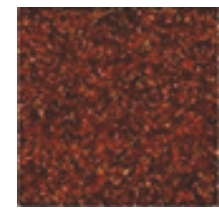

M: 97

5

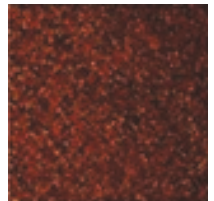

M: 80

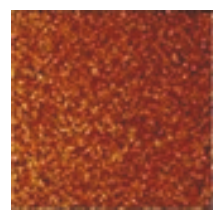

M: 93

6

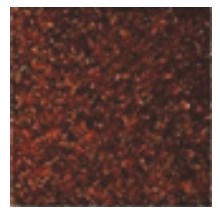

M: 97

6

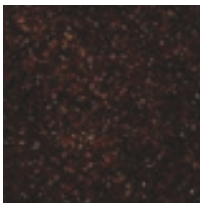

M: 80

7

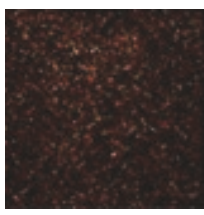

M: 93

7

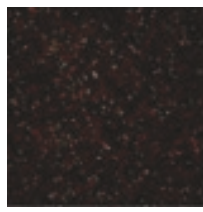

M: 97

7

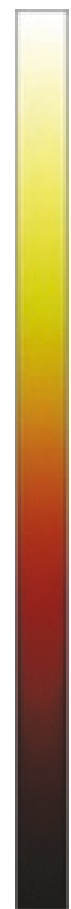

Figure 4: Positive ion TOF-SIMS images of characteristic ions belonging to 4-AMTP $(\mathrm{m} / z=80$, 93, and 97) obtained at positions 1-7 shown in Figure 2.

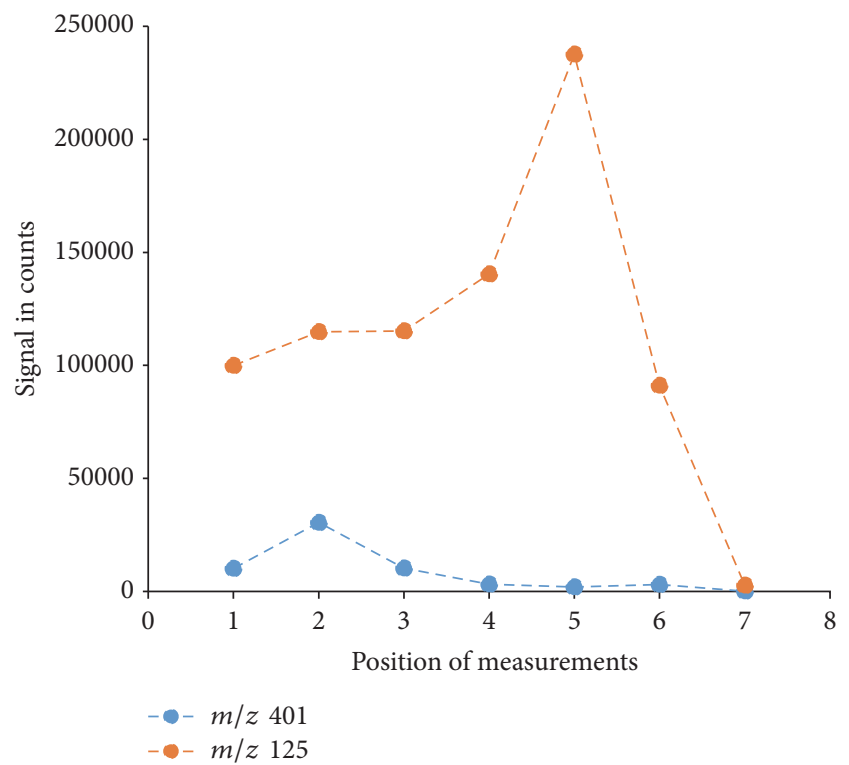

FIgURE 5: Measured TOF-SIMS analyte signal intensities as a function of analyte surface position. The shown TOF-SIMS signal was obtained by summing up the measured counts for the individual molecules over the entire image area.

tested for separation of a simple two-component mixture. The highly uniform nanoscale silver layer favored the analyte fast mass transfer and rapid kinetics. The positions of both preconcentrated molecules could readily be detected with TOF-SIMS, based on molecular and fragment ions. R6G and 4-AMTP were well resolved with the retention factors 0.54 and 0.18. 4-AMTP was irreversibly retained on a silver nanorods surface to form a SAM with "hotspots" with coordinated phenyl molecule. R6G moved over the AMTPSAM as well as the silver nanorods surface. The mechanism of separation is unknown. The analytes were driven differently due to the behavior of nanostructured surface and solvent applied. The data clearly show that this approach could lead to the preparation of functional nanostructured films and their integration into the miniaturized systems to form functional nanodevices.

\section{Conflicts of Interest}

The authors declare that they have no conflicts of interest.

\section{Acknowledgments}

This research has been financially supported by Grants MŠ SR VEGA 1/0074/17 and APVV-16-0029. For a partial research, the infrastructure of the SIX Center was used.

\section{References}

[1] S. A. Archer-Hartmann, L. A. Holland, and R. E. Majors, "Selfassembled nanomaterials for enhanced chemical separation," LC-GC International, vol. 29, no. 5, pp. 1-7, 2011.

[2] L. W. Bezuidenhout and M. J. Brett, "Ultrathin layer chromatography on nanostructured thin films," Journal of Chromatography A, vol. 1183, no. 1-2, pp. 179-185, 2008. 
[3] J. Z. Hall, M. T. Taschuk, and M. J. Brett, "Polarity-adjustable reversed phase ultrathin-layer chromatography," Journal of Chromatography A, vol. 1266, pp. 168-174, 2012.

[4] A. J. Oko, S. R. Jim, M. T. Taschuk, and M. J. Brett, "Time resolved chromatograms in ultra-thin layer chromatography," Journal of Chromatography A, vol. 1249, pp. 226-232, 2012.

[5] T. B. Ledur Kist and M. Mandaji, "Separation of biomolecules using electrophoresis and nanostructures," Electrophoresis, vol. 25, no. 21-22, pp. 3492-3497, 2004.

[6] R. Xiao, K. H. Chu, and E. N. Wang, "Multilayer liquid spreading on superhydrophilic nanostructured surfaces," Applied Physics Letters, vol. 94, no. 5, p. 193104, 2009.

[7] Ch. L. Choong, W. I. Milne, and K. B. K. Teo, "Review: carbon nanotube for microfluidic lab-on-a-chip application," International Journal of Material Forming, vol. 1, no. 2, pp. 117125, 2008.

[8] L. W. Bezuidenhout, N. Nazemifard, A. B. Jemere, D. J. Harrison, and M. J. Brett, "Microchannels filled with diverse microand nanostructures fabricated by glancing angle deposition," Lab on a Chip - Miniaturisation for Chemistry and Biology, vol. 11, no. 9, pp. 1671-1678, 2011.

[9] N. V. Lavrik, L. T. Taylor, and M. J. Sepaniak, "Nanotechnology and chip level systems for pressure driven liquid chromatography and emerging analytical separation techniques: A review," Analytica Chimica Acta, vol. 694, no. 1-2, pp. 6-20, 2011.

[10] K. B. Mogensen, L. Gangloff, P. Boggild, K. B. K. Teo, W. I. Milne, and J. P. Kutter, "Carbon nanotubes integrated in electrically insulated channels for lab-on-a-chip applications," Nanotechnology, vol. 20, no. 9, Article ID 095503, 2009.

[11] J. B. Edel and A. De Mello, "Nanofluidics nanoscience and nanotechnology," Royal Society of Chemistry, 2009.

[12] M. Lard, L. ten Siethoff, S. Kumar et al., "Ultrafast molecular motor driven nanoseparation and biosensing," Biosensors and Bioelectronics, vol. 48, pp. 145-152, 2013.

[13] J. Chen, J. Abell, Y.-W. Huang, and Y. Zhao, “On-chip ultrathin layer chromatography and surface enhanced Raman spectroscopy," Lab on a Chip, vol. 12, no. 17, pp. 3096-3102, 2012.

[14] W. Ch. Tian, "Microfluidics for biological applications," in Science and Business Media, E. Finehout, Ed., Springer, 2008.

[15] J. C. T. Eijkel and A. van den Berg, "Nanofluidics: What is it and what can we expect from it?" Microfluidics and Nanofluidics, vol. 1, no. 3, pp. 249-267, 2005.

[16] A. Van Oudenaarden and S. G. Boxer, "Brownian ratchets: Molecular separations in lipid bilayers supported on patterned arrays," Science, vol. 285, no. 5430, pp. 1046-1048, 1999.

[17] M. O. Magnasco, "Forced thermal ratchets," Physical Review Letters, vol. 71, no. 10, pp. 1477-1481, 1993.

[18] O. O. Saed, R. Li, and Y. Deng, "Microfluidic approcahes for cancer cell separation," Journal of Biomedical Science and Engineering, vol. 7, no. 12, pp. 1005-1018, 2014.

[19] X. Ding, Z. Peng, S.-C. S. Lin et al., "Cell separation using tilted-angle standing surface acoustic waves," Proceedings of the National Academy of Sciences of the United States of America, vol. 111, no. 36, pp. 12992-12997, 2014.

[20] H. Kynclova, J. Pribyl, and R. Hrdy, "Nanostructured surface effect of electrode on doxorubicin determination," Applied Mechanics and Materials, vol. 490-491, pp. 262-266, 2014.

[21] L. Škantárová, A. Oriňák, R. Oriňáková, and F. Lofaj, “4Aminothiophenol strong SERS signal enhancement at electrodeposited silver surface," Nano-Micro Letters, vol. 4, no. 3, pp. 184-188, 2012.
[22] J. Wannenmacher, S. R. Jim, M. T. Taschuk, M. J. Brett, and G. E. Morlock, "Ultrathin-layer chromatography on $\mathrm{SiO} 2$, $\mathrm{Al} 2 \mathrm{O} 3, \mathrm{TiO} 2$, and $\mathrm{ZrO} 2$ nanostructured thin films," Journal of Chromatography A, vol. 1318, pp. 234-243, 2013.

[23] H. Kalász and M. Báthori, "Present status and future perspectives of thin layer chromatography," LC-GC International, vol. 10, no. 7, pp. 440-444, 1997.

[24] D. S. Jensen, S. S. Kanyal, V. Gupta et al., "Stable, microfabricated thin layer chromatography plates without volume distortion on patterned, carbon and Al2O3-primed carbon nanotube forests," Journal of Chromatography A, vol. 1257, pp. 195-203, 2012.

[25] L. A. Wiest, D. S. Jensen, C.-H. Hung et al., "Pellicular particles with spherical carbon cores and porous nanodiamond/polymer shells for reversed-phase HPLC, Analytical Chemistry, vol. 83, no. 14, pp. 5488-5501, 2011.

[26] S. S. Kanyal, D. S. Jensen, A. J. Miles et al., "Effects of catalyst thickness on the fabrication and performance of carbon nanotube-templated thin layer chromatography plates," Journal of Vacuum Science and Technology B:Nanotechnology and Microelectronics, vol. 31, no. 3, Article ID 031203, 2013.

[27] D. S. Jensen, S. S. Kanyal, N. Madaan et al., "Multi-instrument characterization of the surfaces and materials in microfabricated, carbon nanotube-templated thin layer chromatography plates. An analogy to 'The Blind Men and the Elephant', Surface and Interface Analysis, vol. 45, no. 8, pp. 1273-1282, 2013.

[28] T.-D. Dang, Y. H. Kim, J. H. Choi, and G.-M. Kim, "A novel simple preparation method of a hydrogel mold for PDMS micro-fluidic device fabrication," Journal of Micromechanics and Microengineering, vol. 22, no. 1, Article ID 015017, 2012.

[29] J. Parisi, L. Su, and Y. Lei, "In situ synthesis of silver nanoparticle decorated vertical nanowalls in a microfluidic device for ultrasensitive in-channel SERS sensing," Lab on a Chip Miniaturisation for Chemistry and Biology, vol. 13, no. 8, pp. 1501-1508, 2013. 

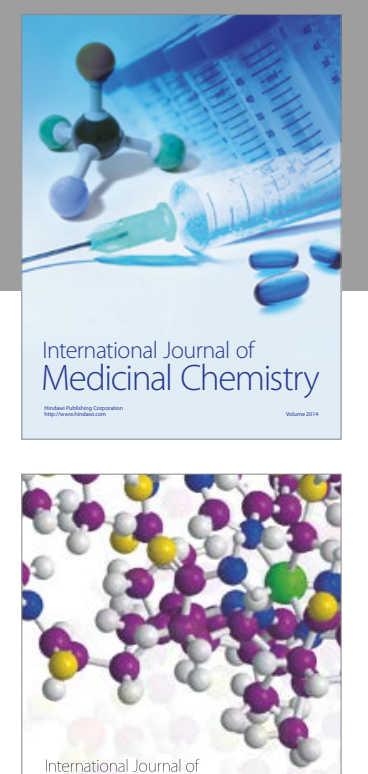

Carbohydrate Chemistry

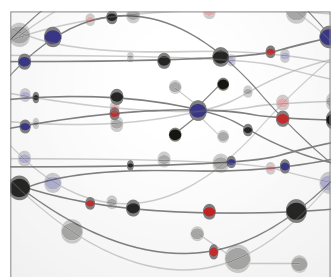

The Scientific World Journal
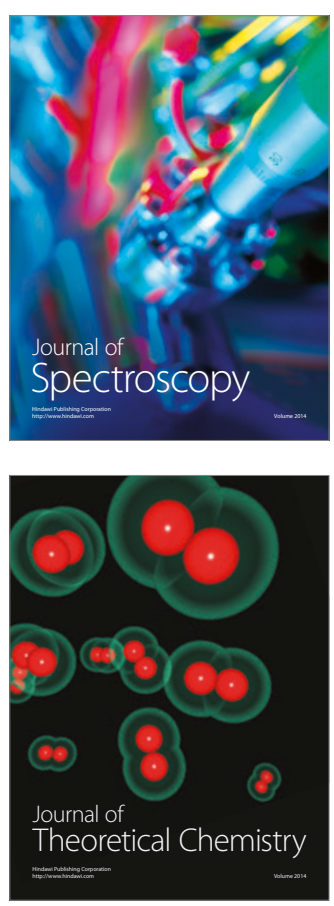
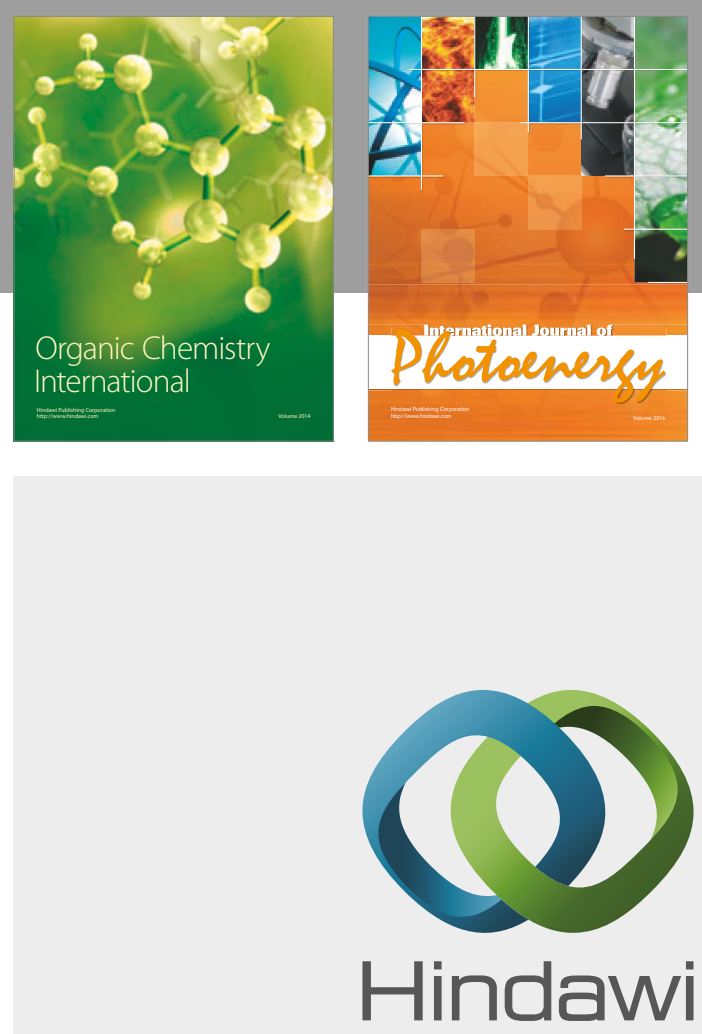

Submit your manuscripts at

https://www.hindawi.com

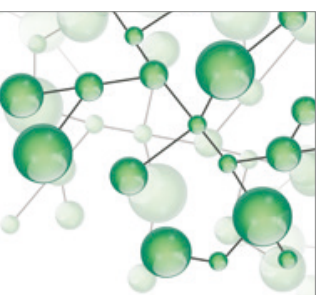

International Journal of

Inorganic Chemistry

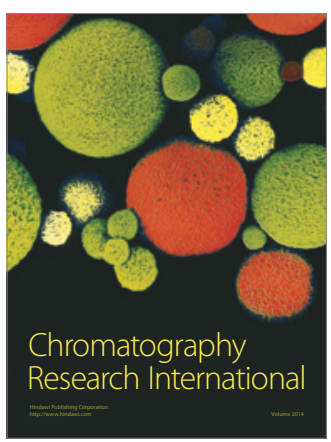

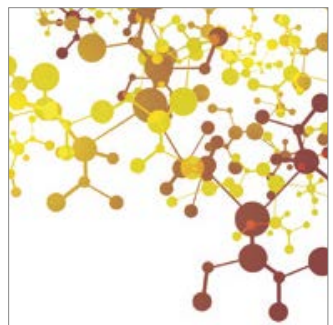

Applied Chemistry
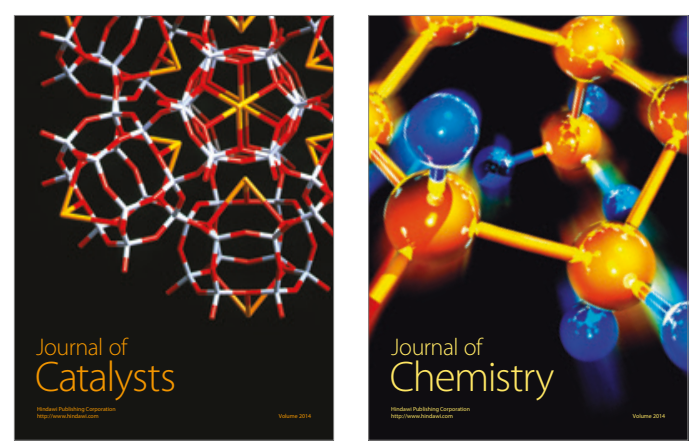
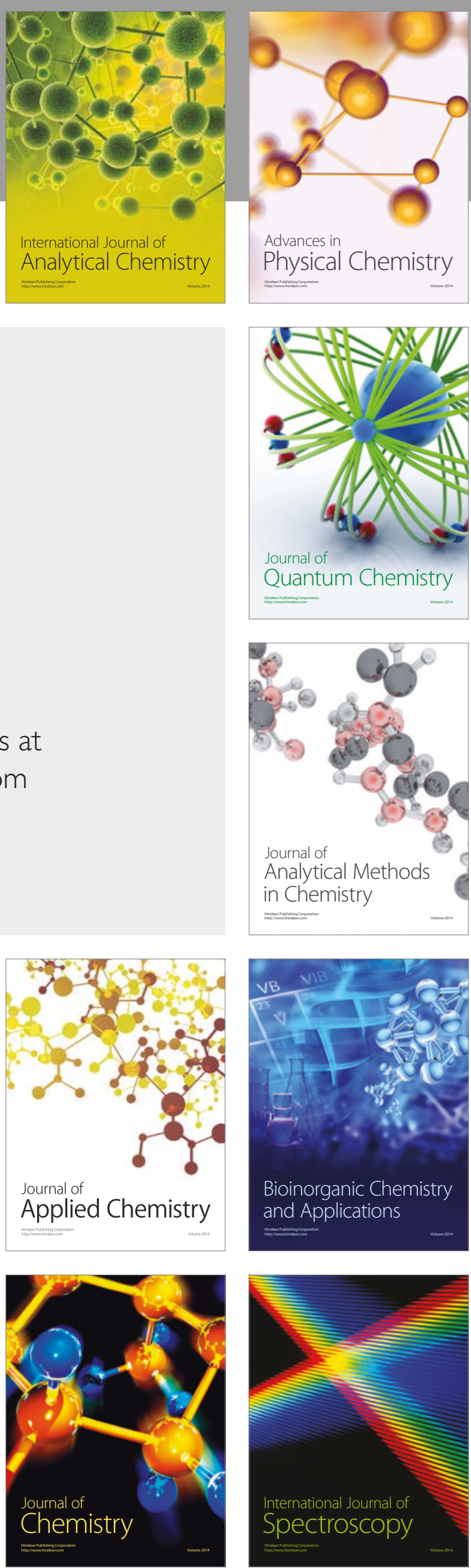